\title{
Minimally invasive decompression for lumbar stenosis and disc herniation
}

\author{
Sean S. Armin, M.D., Langston T. Holly, M.D., and Larry T. Khoo, M.D. \\ Department of Neurosurgery, Comprehensive Spine Center, University of California Los Angeles, \\ Santa Monica, California
}

\begin{abstract}
For decades, lumbar disc herniation and lumbar stenosis have been treated surgically via traditional open techniques. With recent emphasis on minimally invasive approaches in spine surgery, a number of new techniques has been introduced that are aimed at treating these 2 common pathological conditions. Currently the most widely used and efficacious minimally invasive technique for treating these disorders is direct decompression with minimally invasive surgery. Due to the scarcity of large randomized studies, however, it is difficult to compare the effectiveness and possible superiority of this technique with traditional decompression. Further studies are needed to evaluate this issue. (DOI: 10.3171/FOC/2008/25/8/E11)
\end{abstract}

\section{KEY WORDS - lumbar decompression - lumbar disc herniation • minimally invasive surgery $\bullet$ spinal stenosis}

$\mathrm{L}$

UMBAR discectomy and lumbar decompression for stenosis are arguably 2 of the most common and long-standing operations in neurosurgery and spine surgery. Although the pathophysiological mechanisms and patient population of these disease entities differ, they both essentially involve compression of the neural elements located in the thecal sac and traversing/exiting nerve roots. Vascular ischemia of nerve roots is also thought to be involved in the pathogenesis of neurogenic claudication in lumbar stenosis. ${ }^{1}$ The compressive elements include herniated discs ventrally, and/or ligamentum flavum and facet hypertrophy dorsally. Despite decades of advances in spine surgery, the gold standard for treatment of both disorders remains direct decompression of the neural elements by removing the herniated portion of the intervertebral disc, bone structures including the lamina and medial facet, and ligamentum flavum, which together can cause central and lateral recess stenosis., ${ }^{4,12}$

More than 70 years ago, Mixter ${ }^{9}$ and Barr (as detailed in Robinson ${ }^{13}$ ) described the technique of lumbar discectomy in detail, and various modifications were made to their approach subsequently. With the advent of the operating microscope, microsurgical procedures for discectomy were then introduced 40 years later by Yaşargil, Caspar, and Williams. ${ }^{20}$ Despite the fact that decades have passed, lumbar microdiscectomy as described by these pioneers has not changed significantly and is still considered the treatment

Abbreviation used in this paper: MIS = minimally invasive surgery. of choice for patients who require surgery for symptomatic lumbar disc herniation after conservative treatment has failed. Similarly, lumbar laminectomy as described decades ago remains the gold standard for surgical management of lumbar stenosis. ${ }^{12}$

Traditional treatment of both disease entities requires a midline lumbar incision, after which the paraspinous muscles are detached from the spinous processes and lamina, and are retracted laterally and often widely, especially in lumbar stenosis. The extent of removal of posterior spinal elements, including interspinous/supraspinous ligaments, facets, lamina, and spinous processes varies based on the severity of spinal compression. Compared to lumbar disc herniation, in which the compressive lesion is usually focal, traditional treatment of spinal stenosis, especially in the case of combined central and lateral recess stenosis, often necessitates significant disruption of posterior spinal elements. It has been suggested that extensive resection of the posterior bone and muscular structures leads to increased postoperative pain, intraoperative blood loss, and morbidity. Extensive paraspinal muscle detachment from the midline osseous elements can cause weakness secondary to muscle denervation. Damage to the "tension band" consisting of the supra- and interspinous ligaments may lead to increased instability with lumbar flexion. ${ }^{18,19}$ "Failed back syndrome" and intractable pain may thus follow. ${ }^{15,16}$ Wide decompressions that destabilize the facet complexes have been associated with worse outcomes. Prolonged postoperative recovery time, especially in the feeble elderly population that comprises the majority of patients with spinal stenosis, can increase medical morbidity related to deep 
TABLE 1

Evolution of invasive treatment for lumbar disc herniation

\begin{tabular}{|c|c|c|}
\hline Technique & History & References \\
\hline lumbar microdiscectomy/microsurgical discectomy & $\begin{array}{l}\text { introduced when operating microscope was invented (1978); modification of lumbar } \\
\text { discectomy: smaller incision/dissection needed }\end{array}$ & 20 \\
\hline percutaneous treatment of lumbar disc herniation & $\begin{array}{l}\text { eliminates nerve root compression by removing portion of central nucleus pulposus; } \\
\text { can't be used for focal disc disease/extruded disc fragments; limited to patients w/ } \\
\text { central disc herniation \& intact anulus }\end{array}$ & 4 \\
\hline automated percutaneous discectomy & 1980s; mechanical removal of central disc; less effective than direct decompression & 6,11 \\
\hline laser-assisted percutaneous discectomy & 1980s; uses laser energy for central discectomy; less effective than direct decompression & 2,4 \\
\hline
\end{tabular}

venous thrombosis, pulmonary embolism, pneumonia, urinary tract infections, ileus, and prolonged narcotic use. ${ }^{1}$

The modern era of various surgical specialties seeks to foster new techniques that minimize tissue trauma without compromising the goals of the specific operation. ${ }^{12}$ Current trends in surgery favor minimally invasive yet maximally effective operative techniques in the hopes of limiting surgery-related morbidity and mortality. Spine surgery is one subspecialty in which minimally invasive techniques are constantly being developed and modified.

Some of the MIS techniques introduced to treat lumbar disc herniation include chemonucleolysis, automated percutaneous lumbar discectomy, modified nucleotomy, and transforaminal endoscopic techniques (Table 1). Overall these techniques seem to be inferior to the traditional gold standard microsurgical approach. Laser discectomy performed using MIS is not able to address extruded or sequestered disc fragments. None of the techniques mentioned can treat lateral recess or foraminal stenosis, hypertrophy of the ligamentum flavum, or osteophytes, which occur in lumbar stenosis., ${ }^{4,8}$

Although a number of different MIS techniques for treatment of lumbar disc herniation (and to a lesser extent for

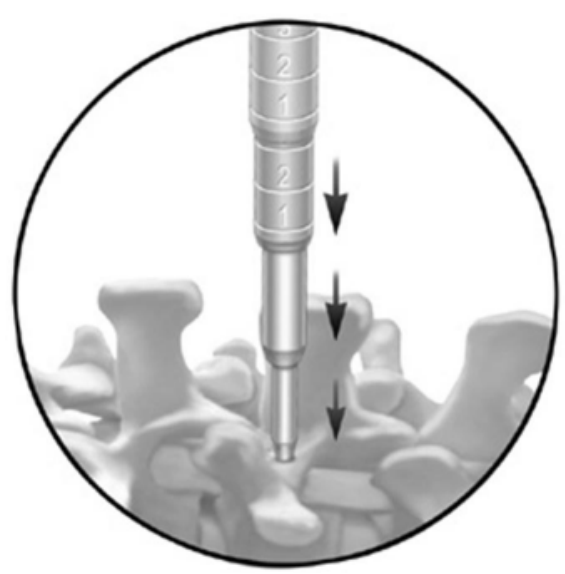

FIG. 1. Schematic demonstrating serial tubular dilators used for muscle splitting and retraction in MIS direct lumbar decompression. lumbar stenosis) have been devised over the past few decades, the direct posterior MIS decompression method seems to be the most widely used technique. This approach, which is a variation on a theme, seeks to take advantage of the time-proven efficacy of direct spinal decompression, while applying a new paradigm for handling soft tissues. The MIS direct lumbar decompression/discectomy favors a "muscle splitting" rather than a subperiosteal dissection technique as is used in traditional methods. In the former technique, a lateral rather than a midline skin incision is used and the paraspinous muscle is not detached from the spinous processes as in traditional techniques. ${ }^{12}$ Instead, the paraspinous muscle fibers are split to provide a corridor to the spinal column. This muscle-splitting method is thought to cause less injury to the soft tissues and thus less postoperative pain. The introduction of various retractors, including tubular corridors (Fig. 1) such as the METRx system (Fig. 2), combined with the operating microscope or the microendoscope, and the aid of fluoroscopy for more accurate localization of the pathological entity to be treated have allowed for a decrease in the size of the skin incision and in the extent of soft-tissue trauma. Because most neurosurgeons are more familiar with the operating microscope, the microendoscope is not as widely used. . $^{1,12}$

In the case of lumbar disc herniation or unilateral lateral recess stenosis, the paraspinal skin incision (usually $1.5 \mathrm{~cm}$ off the midline for lateral recess stenosis/paracentral disc herniation; $>3 \mathrm{~cm}$ off midline for far-lateral disc herniation) is made ipsilateral to the disc to allow a more direct path for discectomy. For treatment of central stenosis, a lateral incision is made on either side of midline but still

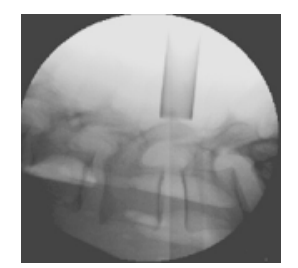

FIG. 2. Fluoroscopic view of the Medtronic METRx working tube docked on the lumbar lamina-facet junction at the level of the disc space. This location can be used for direct posterior bone/ligamentous decompression as well as microdiscectomy. 


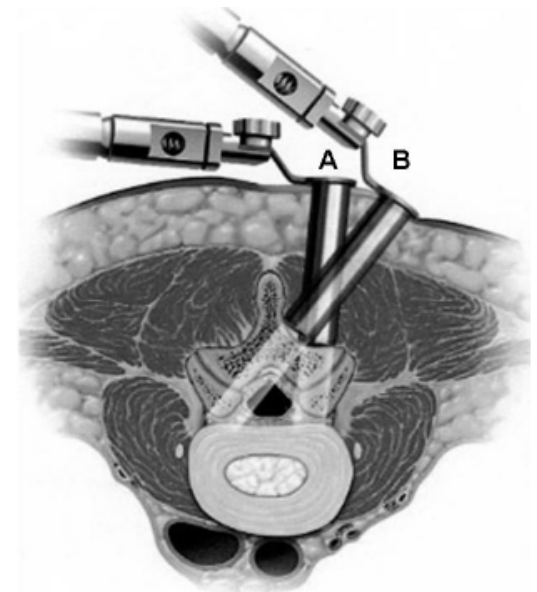

FIG. 3. Schematic showing the Medtronic METRx tubular retractor locked in 2 different positions. A: Lateral position over the lamina-facet junction ideal for ipsilateral decompression of lateral recess stenosis or microdiscectomy. B: Medial position over the lamina-spinous process junction ideal for decompression of central canal and partial contralateral lateral recess.

allows for bilateral decompression, while preserving the spinous processes and midline tension band (Fig. 3). This approach takes advantage of modifications previously made to the traditional decompression method, in which

TABLE 2

Characteristics of group undergoing MIS in lumbar stenosis decompression study*

\begin{tabular}{lr}
\hline \multicolumn{1}{c}{ Characteristic } & \multicolumn{1}{c}{ Value } \\
\hline total no. of patients & 48 \\
mean age & 64.5 \\
male/female ratio & $3: 2$ \\
presenting symptom (\% of patients) & 100 \\
low-back pain & 80 \\
lower-extremity radiculopathy & 96 \\
gait difficulty & 16 \\
urinary dysfunction & \\
extent of decompression & 28 patients \\
1 level & 20 patients \\
2 levels & $4 \% \dagger$ \\
intraop CSF leak & none \\
intraop neurological injury & \\
postop lumbar instability & \\
clinical outcome (32 of 48 patients) & 3 -yr follow-up \\
ODI score & 46 \\
preop & 20 \\
1 yr postop & 21 \\
2 yrs postop & 26 \\
3 yrs postop & \\
SF-36 score & \\
preop & 2.2 \\
1 yr postop & 3.1 \\
2 yrs postop & 2.9 \\
3 yrs postop & 3 \\
\hline
\end{tabular}

* Reported by Asgarzadie and Khoo. Exclusion criteria included need for lumbar decompression combined with fusion, evidence of lumbar instability/severe deformity/spondyloptosis/severe spondylolisthesis, infection, tumor, or cauda equina syndrome. Abbreviations: ODI = Oswestry Disability Index; SF-36 = Short Form Health Survey.

$\dagger$ Up to $16 \%$ in previous literature for MIS decompression (see Khoo and Fessler). bilateral hemilaminotomy is performed while minimizing muscle trauma. In the case of bilateral symptomatic lateral recess stenosis, paraspinal incisions may need to be made on both sides to allow for adequate decompression. Similarly, a far-lateral disc herniation with concurrent central stenosis must either be treated by way of 2 separate paraspinal incisions or an open technique. ${ }^{1}$

To our knowledge, there are no rigorous data available that compare the postsurgical outcome of traditional microdiscectomy versus muscle-splitting microdiscectomy. Schizas et al. ${ }^{14}$ report on 28 patients with a lumbar disc herniation, of whom half underwent traditional microdiscectomy and the other half underwent microendoscopic discectomy. Although there was no difference in pain outcomes between the 2 groups at the 1 -year follow-up review, the patients in the latter group required 8.8 doses of postoperative oral analgesics compared with 12 doses in the former group (a $26 \%$ difference). Although this study seems to support the notion that the muscle-splitting method causes less postoperative pain, large prospective randomized studies with long-term follow-up are still lacking.

Similarly, MIS techniques when used for direct bilateral decompression of lumbar stenosis via a unilateral approach have been shown to offer a similar short-term clinical outcome when compared with traditional techniques, while decreasing intraoperative blood loss, postoperative stay, and use of narcotics. ${ }^{7}$ The microendoscopic decompressive laminotomy technique was also validated in a series of cadaveric studies in which equivalent bone decompressions were obtained using either the traditional or endoscopic technique. ${ }^{5}$ Asgarzadie and $\mathrm{Khoo}^{1}$ compare 48 patients with central canal stenosis with or without lateral recess stenosis who were treated by MIS decompression (Table 2) with a historical cohort of 32 patients who were treated by traditional decompression. The average length of hospital stay for the former and latter groups was 36 and 94 hours, respectively. All 32 of the 48 patients in the MIS group who were followed for 4 years showed an increase in walking endurance at 6 months, and $88 \%$ of those who were followed up for 4 years (78\% of patients) reported improved symptoms.

In patients with lumbar stenosis in the setting of spondylolisthesis, scoliosis, or severe degenerative disc disease, the inherent destabilizing nature of posterior decompression, even using MIS methods, may warrant a fusion operation in addition to decompression. The presence of severe spondylolisthesis or deformity, infection, tumor, arachnoiditis, pseudomeningocele, or a cerebrospinal fluid fistula makes MIS decompression for lumbar stenosis less desirable. Redoing MIS decompression is technically difficult, and is associated with increased risk for durotomy and for conversion to an open (traditional) technique. ${ }^{1}$

Although small nonrandomized studies as mentioned earlier do suggest a marginally smaller postoperative oral analgesic requirement and decreased length of hospital stay in the MIS direct decompression group, the actual efficacy and suspected superiority of the MIS technique compared to the traditional technique is still not well characterized. Future studies are still needed to determine the role of the MIS method in the treatment of lumbar stenosis and disc herniation. 


\section{Disclaimer}

The authors report no conflict of interest concerning the materials or methods used in this study or the findings specified in this paper.

\section{References}

1. Asgarzadie F, Khoo LT: Minimally invasive operative management for lumbar spinal stenosis: overview of early and long-term outcomes. Orthop Clin North Am 38:387-399, 2007

2. Choy DS, Ascher PW, Ranu HS, Saddekni S, Alkaitis D, Liebler W, et al: Percutaneous laser disc decompression. A new therapeutic modality. Spine 17:949-956, 1992

3. Dandy WE: Loose cartilage from intervertebral disk simulating tumor of the spinal cord. By Walter E. Dandy, 1929. Clin Orthop Relat Res 238:4-8, 1989

4. Deen HG, Fenton DS, Lamer TJ: Minimally invasive procedures for disorders of the lumbar spine. Mayo Clin Proc 78: 1249-1256, 2003

5. Guiot BH, Khoo LT, Fessler RG: A minimally invasive technique for decompression of the lumbar spine. Spine 27:432-438, 2002

6. Kahanovitz N, Viola K, Goldstein T, Dawson E: A multicenter analysis of percutaneous discectomy. Spine 15:713-715, 1990

7. Khoo LT, Fessler RG: Microendoscopic decompressive laminotomy for the treatment of lumbar stenosis. Neurosurgery 51 (5 Suppl):S146-S154, 2002

8. Maroon JC: Current concepts in minimally invasive discectomy. Neurosurgery 51 (5 Suppl):S137-S145, 2002

9. Mixter WJ: Rupture of the lumbar intervertebral disk: an etiologic factor for so-called "sciatic" pain. Ann Surg 106:777-787, 1937

10. Nordby EJ, Wright PH: Efficacy of chymopapain in chemonucleolysis. A review. Spine 19:2578-2583, 1994

11. Onik G, Mooney V, Maroon JC, Wiltse L, Helms C, Schweigel J, et al: Automated percutaneous discectomy: a prospective multiinstitutional study. Neurosurgery 26:228-233, 1990
12. Riesenburger RI, David CA: Lumbar microdiscectomy and microendoscopic discectomy. Minim Invasive Ther Allied Technol 15:267-270, 2006

13. Robinson JS: Sciatica and the lumbar disk syndrome: a historic perspective. South Med J 76:232-238, 1983

14. Schizas C, Tsiridis E, Saksena J: Microendoscopic discectomy compared with standard microsurgical discectomy for treatment of uncontained or large contained disc herniations. Neurosurgery 57 (4 Suppl):357-360, 2005

15. See DH, Kraft GH: Electromyography in paraspinal muscles following surgery for root compression. Arch Phys Med Rehabil 56:80-83, 1975

16. Sihvonen T, Herno A, Paljärvi L, Airaksinen O, Partanen J, Tapaninaho A: Local denervation atrophy of paraspinal muscles in postoperative failed back syndrome. Spine 18:575-581, 1993

17. Smith L: Enzyme dissolution of the nucleus pulposis in humans. JAMA 187:137-140, 1964

18. Tsai RY, Yang RS, Bray RS Jr: Microscopic laminotomies for degenerative lumbar spinal stenosis. J Spinal Disord 11: 389-394, 1998

19. Tuite GF, Stern JD, Doran SE, Papadopoulos SM, McGillicuddy JE, Oyedijo DI, et al: Outcome after laminectomy for lumbar spinal stenosis. Part I: clinical correlations. J Neurosurg 81: 699-706, 1994

20. Williams RW: Microlumbar discectomy: a conservative surgical approach to the virgin herniated lumbar disc. Spine 3:175-182, 1978

Manuscript submitted April 16, 2008.

Accepted June 24, 2008.

Address correspondence to: Larry T. Khoo, M.D., Division of Neurosurgery, Comprehensive Spine Center, University of California Los Angeles, 1245 16th Street, Suite 220, Santa Monica, California 90404. email: 1khoo@mednet.ucla.edu. 